\title{
Femtosecond Laser Processing of Polymethyl Methacrylate with an Axicon
}

\author{
Yu Matushiro and Wataru Watanabe \\ ${ }^{* 1}$ Department of Electrical \& Electronic Engineering, College of Science and Engineering, \\ Ritsumeikan University \\ 1-1-1 Nojihigashi, Kusatsu, Shiga, 525-8577 Japan \\ E-mail: wata-w@fc.ritsumei.ac.jp
}

\begin{abstract}
Focusing a laser beam with an axicon lens produces a Bessel beam and increases the longitudinal interaction length. This report describes structural modifications to be extended deep in polymethyl methacrylate (PMMA) using a femtosecond laser with an axicon. We investigated the dependence of the morphology of filamentary structural modifications on the distance between the tip of axicon and the sample surface. The region of filamentary modification was greater than $10 \mathrm{~mm}$ long. Focusing-refocusing modifications of filaments were produced.
\end{abstract}

DOI: $10.2961 / \mathrm{jlmn} .2016 .01 .0011$

Keywords: femtosecond laser, micromachining, axicon, Bessel beam, filamentation, polymer

\section{Introduction}

The use of femtosecond laser direct writing inside transparent materials has been growing rapidly. Tightly focused femtosecond laser pulses can induce structural modifications around the focal volume in transparent materials [1-4] When a femtosecond laser pulse is focused loosely inside the bulk of a transparent material, filamentation occurs as the result of a dynamic balance between Kerr self-focusing and defocusing effects in the electron plasma that is generated through the ionization process. The electron plasma intensity in the filamentary region can become sufficiently high to produce localized modification in the filamentary volume. [5-8]. Induction of filamentary refractive-index change, a promising technique for use in internal micromachining, has been applied widely to fabrication of optical elements such as waveguides and gratings in glass [1-4] and polymer materials [9-19]. For internal micromachining inside transparent materials, Gaussian laser beams are commonly focused using objective lenses. However, the processing range of structural modification is limited even if filamentation occurs. This limitation does not accommodate full exploitation of the three-dimensional capabilities of femtosecond laser micromachining.

To overcome such limitations, application of nondiffractive Bessel beams has been attempted for laser materials processing $[20,21]$. Because the intensity maximum is strongly localized over a distance that exceeds the Rayleigh range of Gaussian beams by orders of magnitude, Bessel beams have attracted great interest for diverse applications. Bessel beams can be generated using an annular slit combined with a lens, computer-generated holograms with spatial light modulators, and axicon lenses [22-26].

Results demonstrated that Bessel beams can provide important benefits for femtosecond laser propagation and processing. Bessel beams have focal depths that are much greater than those of Gaussian beams with a combination of diffraction-free nature of Bessel beam and nonlinear propagation such as filamentation. Especially, focusing of femto- second pulse axicons provides distinct benefits because longer plasma channels can be formed than in the case of a lens focusing a Gaussian beam in air [27,28], or liquid $[29,30]$ or solid $[31,32]$ matter. In glass, modifications or damage tracks were found over longer distances [33-35]. For femtosecond laser micromachining, Bessel beams have been used for surface ablation [36,37], channel formation [38,39], and writing waveguides [40] and gratings [41] within bulk glasses, and for glass cutting [42].

As described herein, we used an axicon lens with a femtosecond laser to study the morphology of structural modifications produced in bulk polymethyl methacrylate (PMMA). This report describes the induction of structural modifications by changing (i) the distance between the PMMA sample and the tip of the axicon and (ii) laser energies. Structural modifications are extended deep into PMMA by a femtosecond laser with an axicon.

\section{Bessel beam propagation with an axicon lens}

When a Gaussian beam is incident on an axicon lens, the transmitted beam after the axicon is called a GaussianBessel beam. The intensity distribution of a GaussianBessel beam in a linear regime is described by a zerothorder Bessel function of the first kind $J_{0}[23]$ as

$$
\begin{aligned}
& I(r, z)=2 k \pi\left(\tan ^{2} \alpha\right)(n-1) z I_{0} e^{-2(n-1) z \tan \alpha / \omega_{0}} \\
& \times J_{0}^{2}(k r(n-1) \tan \alpha),
\end{aligned}
$$

where $r$ and $z$ respectively denote the radial and longitudinal coordinates, $I_{0}$ and $\omega_{0}$ respectively represent the intensity and beam waist of the incident Gaussian beam, $k$ is the wave vector, $n$ is the index of refraction of the axicon lens, and $a$ is the wedge angle. The relation between wedge angle $a$ and cone angle $\theta$ is given as

$\alpha=\left(\frac{180-\theta}{2}\right)$.

Figure 1 shows on-axis intensity variation $(r=0)$ along the beam propagation direction $(z)$ for a Gaussian-Bessel beam 
when the laser beam of $800 \mathrm{~nm}$ wavelength and $6 \mathrm{~mm}$ beam diameter is focused by an axicon with refractive index $n$ of 1.51 and cone angle of $170^{\circ}$. The Rayleigh range of a Gaussian-Bessel beam $z_{\mathrm{r}}$ is given as [23]

$Z_{r} \approx \frac{D}{2(n-1) \tan (\alpha)}$

where $D$ denotes the incident laser beam diameter. Using eq. (3), the calculated $z_{\mathrm{r}}$ is $66 \mathrm{~mm}$. From eq. (1), the central core diameter of the Gaussian-Bessel beam is estimated as 6.9 $\mu \mathrm{m}$.

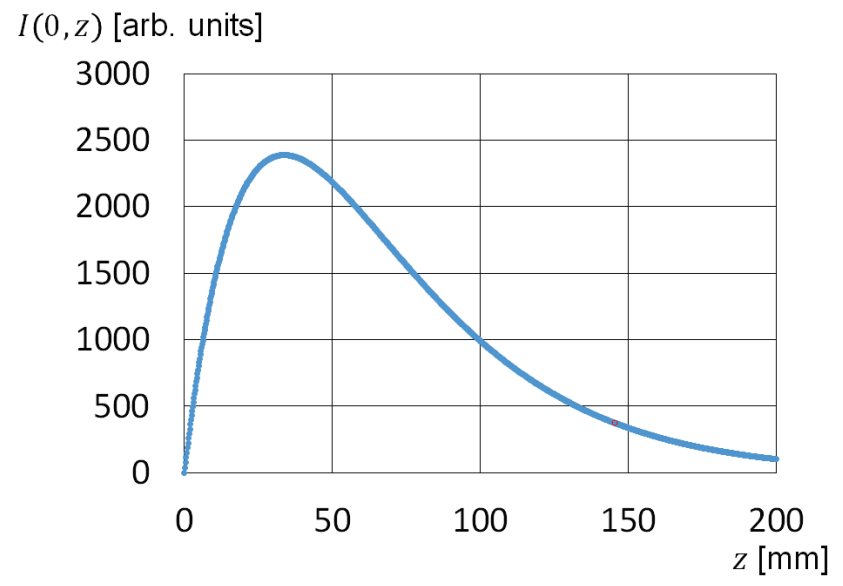

Fig. 1 On-axis intensity variation $(r=0)$ along the beam propagation direction $(z)$ for a Bessel beam created using an axicon.

\section{Experimental system}

Figure 2 shows the optical setup for femtosecond laser processing with an axicon. Laser pulses of 100 fs were generated using a Ti:sapphire laser system with $800 \mathrm{~nm}$ wavelength of, a repetition rate of $1 \mathrm{kHz}$, and maximum laser power of $0.4 \mathrm{~mJ} /$ pulse. The pulse energy was attenuated by rotating a half-wave plate in front of a polarizer. The Gaussian beam impinged on the axicon was $6 \mathrm{~mm}\left(1 / \mathrm{e}^{2}\right)$. The $\mathrm{M}$ square of the laser beam was approximately 1.3. Axicon lenses were used to transform the laser beam from the Gaussian to the Bessel function of intensity distribution. An axicon with cone angle of $170^{\circ}$ was used. The PMMA sample is a 20 $\mathrm{mm}$ cube. The four sides of the sample were optically polished. The sample was mounted on a computer-controlled translation.

Structural modification was observed from the direction perpendicular to the optical axis using a transilluminated optical microscope. Structural modifications or refractive index changes in glass with axicons were observed with optical microscopes at fixed exposure of laser pulses [40 42]. However, the refractive index change in PMMA is on the order of $10^{-4}$, which is lower than those induced in widely various glasses by the order of $10^{-3}$. Therefore, it is difficult to observe structural modifications at fixed exposure. To visualize structural modifications from the side (in the $y$ $z$ plane), the sample was translated perpendicularly ( $x$ axis) to the laser beam propagation direction ( $z$ axis) at a constant speed. Periodic structures with some intervals are written in the PMMA (Fig. 3).

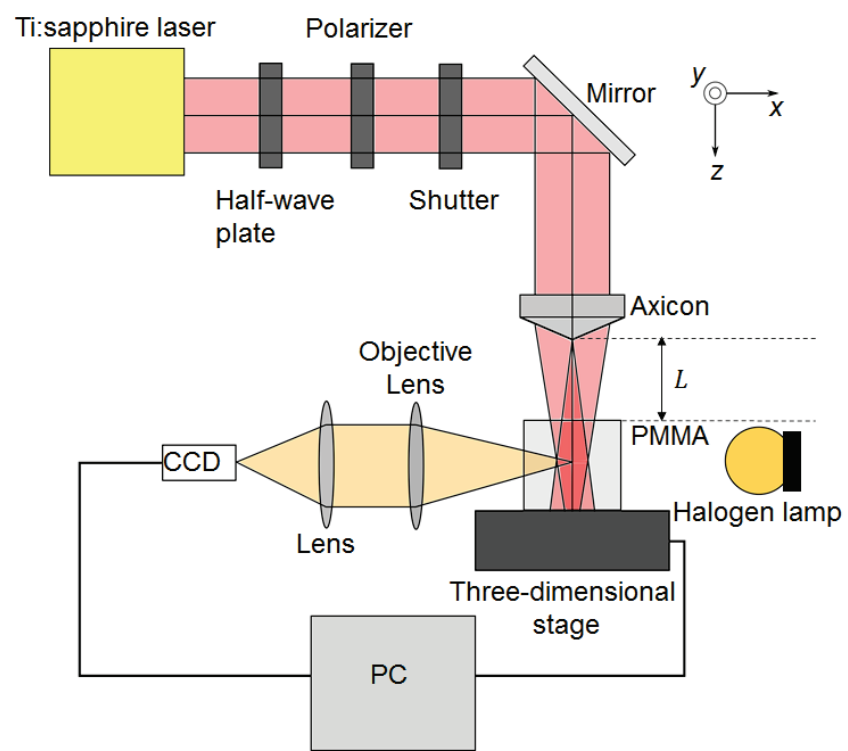

Fig. 2 Optical setup for femtosecond laser processing with an axicon: PC, personal computer; CCD, CCD camera.

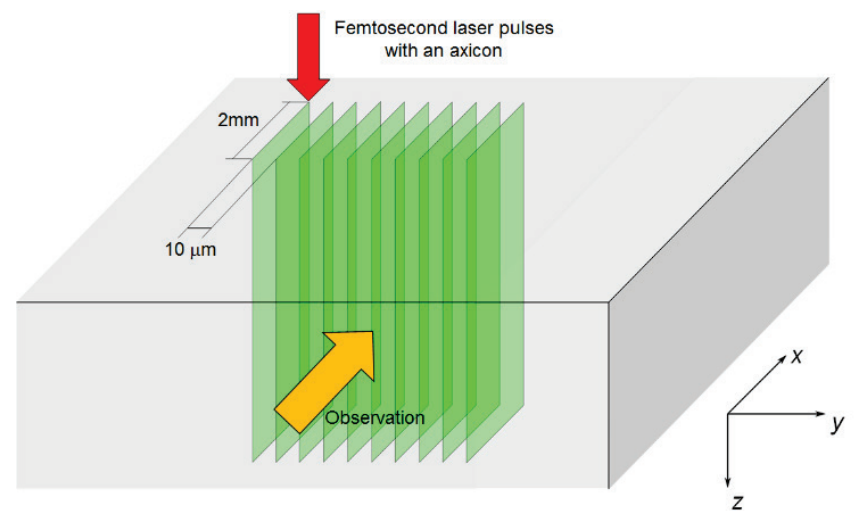

Fig. 3 Schematic diagram for induction of structural modifications in PMMA. To visualize structural modifications from the side, the sample was translated $2 \mathrm{~mm}$ perpendicularly to the laser beam propagation direction at constant speed. Periodic structures with an interval of $10 \mu \mathrm{m}$ are shown.

\section{Experimental results}

4.1 Structural modifications as a function of distance between the PMMA sample and the tip of the axicon

We experimentally studied structural modifications in PMMA. We investigated structural modifications in PMMA by an axicon with a $170^{\circ}$ cone angle by changing the distance between the axicon and the sample surface. Figure 4 shows optical microscopic images of the laser induced structures in PMMA with a femtosecond Bessel beam. Variations of structural modifications for different distance between the axicon tip and sample surface are demonstrated at a constant energy of $0.2 \mathrm{~mJ} /$ pulse and constant speed of 0.5 $\mathrm{mm} / \mathrm{s}$. The sample was translated $2 \mathrm{~mm}$ along the $\mathrm{y}$-axis perpendicular to the laser propagation axis at the speed of $0.5 \mathrm{~mm} / \mathrm{s}$ and repeated 10 times every $10 \mu \mathrm{m}$ ( $x$ axis $)$. At 5 $\mathrm{mm}$ distance, structural modification started at $5 \mathrm{~mm}$ from 
the incident surface and continued up to the back surface. At distances of $15 \mathrm{~mm}, 25 \mathrm{~mm}$, and $35 \mathrm{~mm}$, structural modification started at the incident surface and ended inside. The filamentary modification length decreased as the distance increased. At the entrance surface, ablation occurred. At a distance of $45 \mathrm{~mm}$ and $55 \mathrm{~mm}$, modifications started inside. No evidence exists of ablation at the entrance or exit surfaces. The region of filamentary modification was great- er than $10 \mathrm{~mm}$ long. The positions and lengths of structural modifications in PMMA are dependent on the distance between the axicon and the PMMA surface. From optical images, structural modifications were confirmed to be the result of refractive-index change. The refractive-index region diameter was several micrometers.

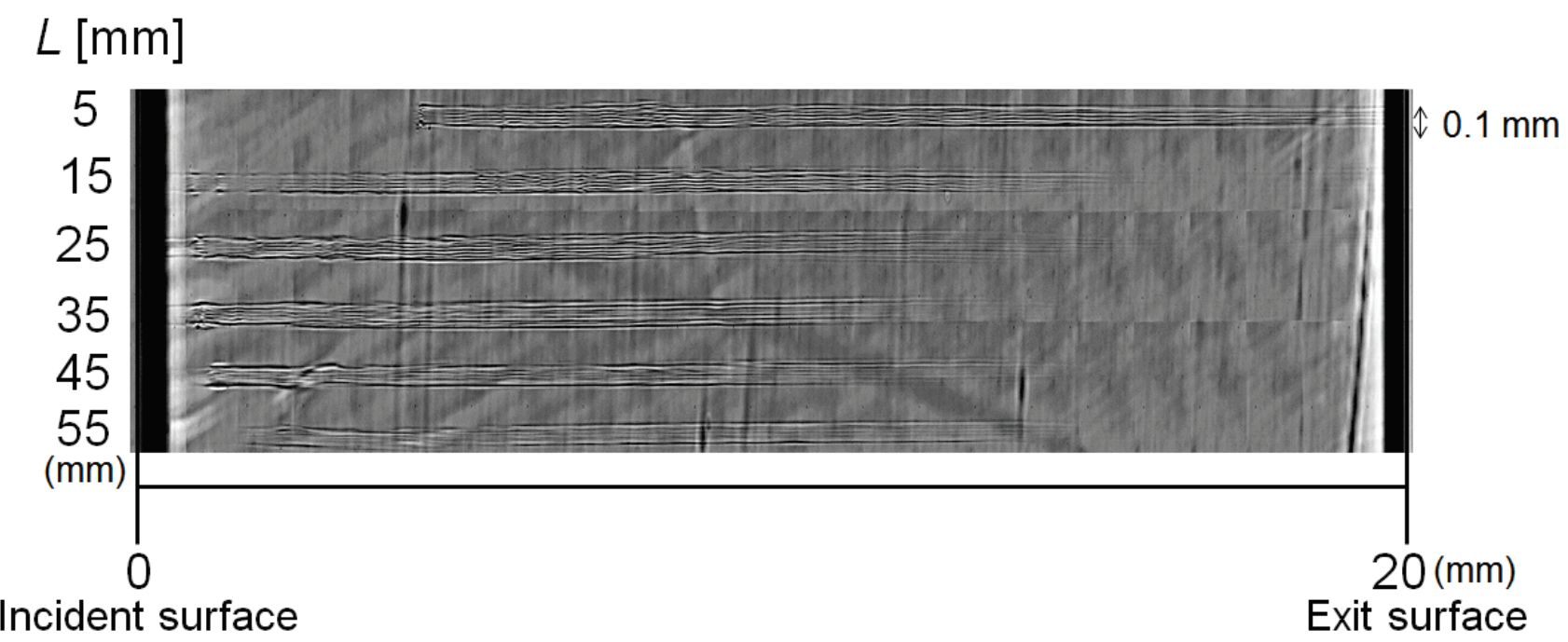

Fig. 4 Optical microscopic images of the laser-induced structures in PMMA with a femtosecond Bessel beam. Variations of structural modifications for different distances between the axicon tip and sample surface from the side. The sample was translated perpendicularly to the optical an axis at $0.5 \mathrm{~mm} / \mathrm{s}$ along the $y$ axis. Translation was repeated 10 times every $10 \mu \mathrm{m}$. The image was obtained by combining images that had been taken separately. The entrance surface of PMMA sample is on the left side. The exit surface is on the right side. The

beam direction is from left to right surface of the sample. The image was observed under illumination using a halogen lamp.

\subsection{Structural modification with different pulse energy}

Figure 5 presents filamentary modifications as a function of the energy of the Bessel beam. Structural modifications were produced at the pulse energy between $0.15 \mathrm{~mJ} /$ pulse, $0.20 \mathrm{~mJ} /$ pulse, and $0.25 \mathrm{~mJ} /$ pulse at a fixed scan speed of $0.5 \mathrm{~mm} / \mathrm{s}$. Figures 5(a) and 4(b) show transmission microscope images of the structural modifications in PMMA with different pulse energy, as observed from a direction perpendicular to the optical axis at a distance of $5 \mathrm{~mm}$ and $25 \mathrm{~mm}$, respectively. Below the pulse energy of $0.1 \mathrm{~mJ} / \mathrm{pulse}$, no structural modification was observed with the optical microscope. Lengths of the modifications along the optical axis increased as the energy increased. Figure 5 also shows that when the pulse energy increased, the head of the filament filamentary track shifts towards the laser entrance. Table 1 presents the head position of the filamentary modi- fications for different pulse energies. We can ascribe this effect to self-focusing of ultrashort pulses [7].

Table 1 Head position of filamentary modifications for different pulse energies

Distance between the PMMA sample and

\begin{tabular}{ccc} 
& \multicolumn{2}{c}{ the axicon tip } \\
\cline { 2 - 3 } $\begin{array}{c}\text { Energy } \\
{[\mathrm{mJ} / \text { pulse }]}\end{array}$ & $5 \mathrm{~mm}$ & $25 \mathrm{~mm}$ \\
\hline 0.15 & 4.7 & 3.5 \\
0.2 & 4.8 & 1.4 \\
0.25 & 5.1 & 0
\end{tabular}




\section{Pulse energy $[\mathrm{mJ} /$ pulse]}

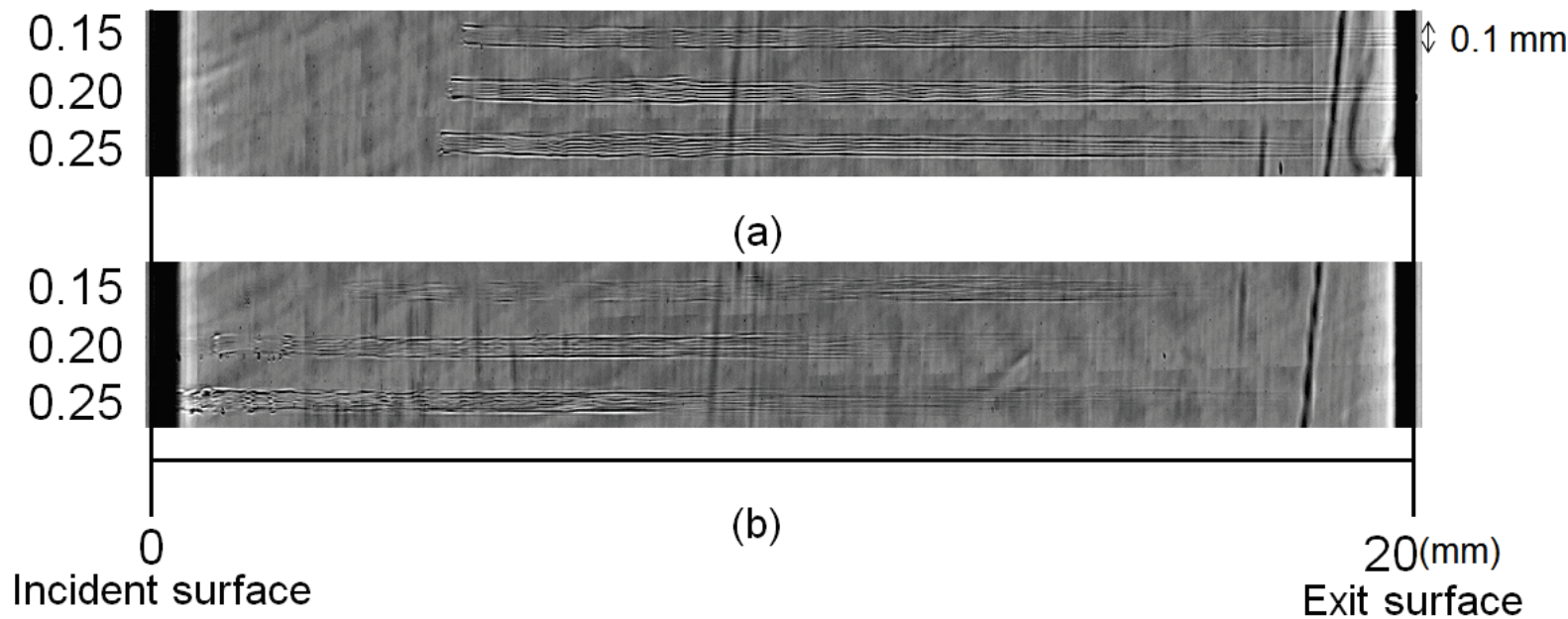

Fig. 5 Variations of structural modifications for different pulse energies. Structural modifications in PMMA were induced at distance $L$ of (a) of 5 and (b) 25 . The sample was translated perpendicularly to the optical an axis at the speed of $0.5 \mathrm{~mm} / \mathrm{s}$ along $y$ axis and was repeated 10 times every $10 \mu \mathrm{m}$.

\subsection{Structural modification with different scan speeds} We investigated the dependence of the region of modifications on scanning speed under fixed pulse energy $(0.2$ $\mathrm{mJ} /$ pulse) at distances of $5 \mathrm{~mm}$ and $25 \mathrm{~mm}$. We changed the scan speed from 0.1 to $1 \mathrm{~mm} / \mathrm{s}$. Figures 6(a) and 6(b) show optical images of the structural modifications in PMMA as a function of the scan speed. The contrast of the microscop- ic image shows features of two types. The black spot in the images is the granular structure or scattering damage. Attempts to translate the sample at lower scanning speed engender the granular structure or scattering damage observed as a result of optical breakdown.

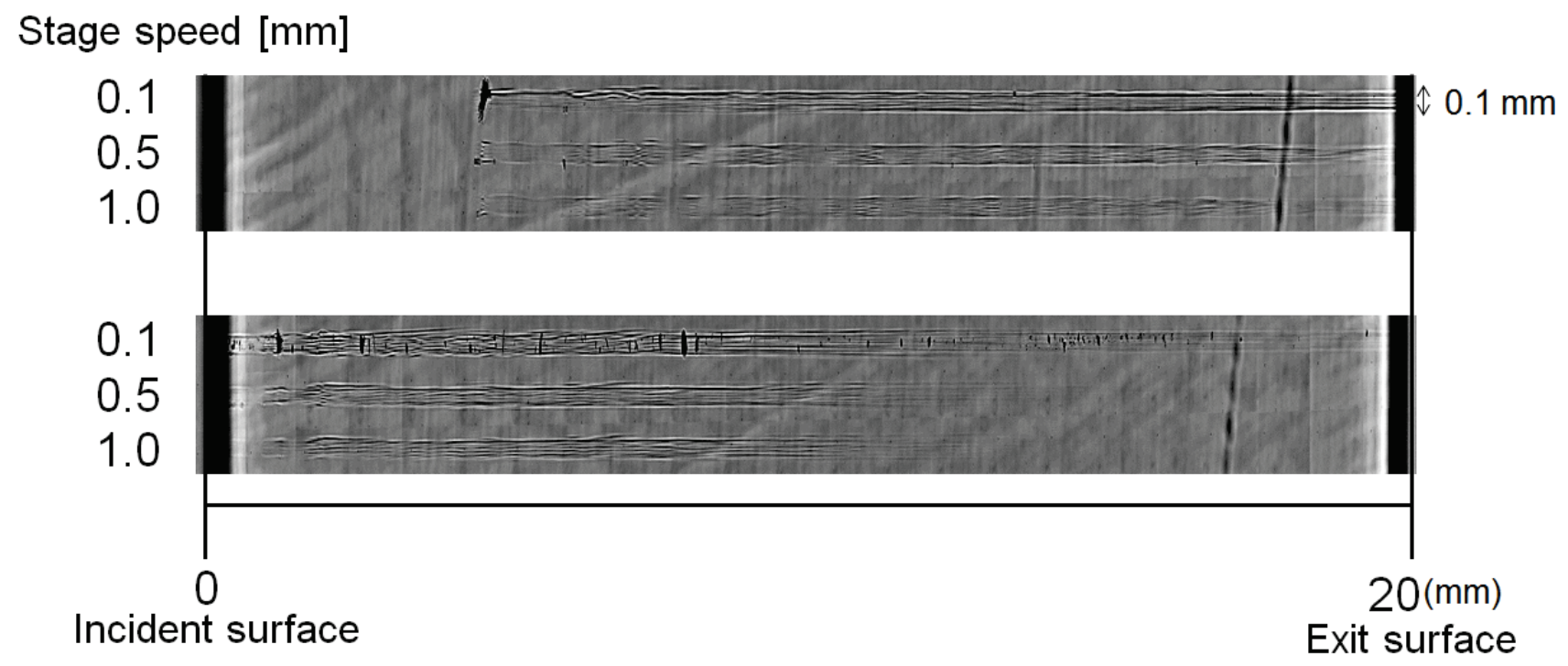

Fig. 6(a) Dependence on scanning speed at distance $r$ of (a) 5 and (b) 25. The sample was translated perpendicularly to the optical axis at different speeds and repeated 10 times every $10 \mu \mathrm{m}$. 


\subsection{Structural modification with different intervals}

To investigate the effects of the written structures, we changed the structure interval. Once a sheet of filamentary modification is induced along the $x$ axis, the next filamentary propagation might be affected by the previously induced region of refractive index change. We changed the interval of structures at a constant energy of $0.2 \mathrm{~mJ} / \mathrm{pulse}$ and a constant speed of $0.5 \mathrm{~mm} / \mathrm{s}$. The distance between the axicon and the sample surface was set as $5 \mathrm{~mm}$. The sample was translated $2 \mathrm{~mm}$ perpendicular to the laser propagation axis. Figure 7(a) presents optical images obtained when the sample was translated perpendicularly to the optical axis every $10 \mu \mathrm{m}$ and $25 \mu \mathrm{m}$, and $50 \mu \mathrm{m}$. Single filamentary modification was induced as a trial at an interval of $50 \mu \mathrm{m}$. Single filamentary modification is a refractive-index change. At the beginning of the modification, a filament breaks into multiple filaments at all the energies. Figure 7(b) presents a magnified image of typical multiple filamentary modifications. Some multiple filamentary modifications disappeared or were combined into one filamentary modification. The complex filamentary modifications are unaffected by previously induced written structures.

Figure 8(a) presents variations of structural modifications for different pulse energies at the distance $L=5 \mathrm{~mm}$ and the interval of $50 \mu \mathrm{m}$. Figure 8(b) shows a magnified image of focusing and refocusing of filamentary modifications. In Fig. 8, focusing-refocusing events are recognizable. At lower energy, the number of focusing-refocusing events increased. After multiple filaments occurred, and focusing and refocusing events were repeated, a single filament with a long channel is formed. Finally, focusing and refocusing events were visible at the end of modifications.

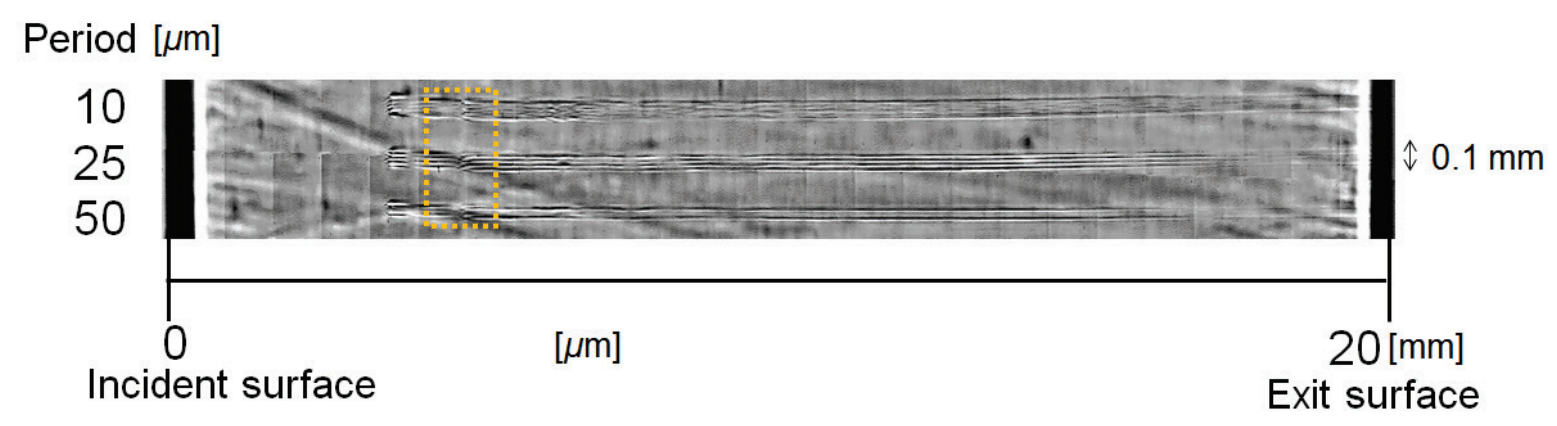

(a)

\section{Period}

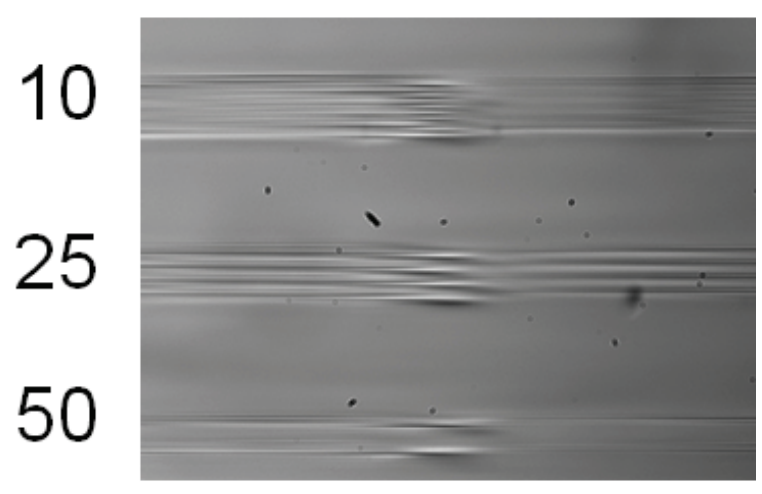

(b)

Fig. 7(a) Dependence on periods at distance $L=5 \mathrm{~mm}$, energy of $0.2 \mathrm{~mJ} /$ pulse, and the speed of $0.5 \mathrm{~mm} / \mathrm{s}$. The sample was translated perpendicularly to the optical axis. (b) Magnified image of multi-filamentary modifications. 


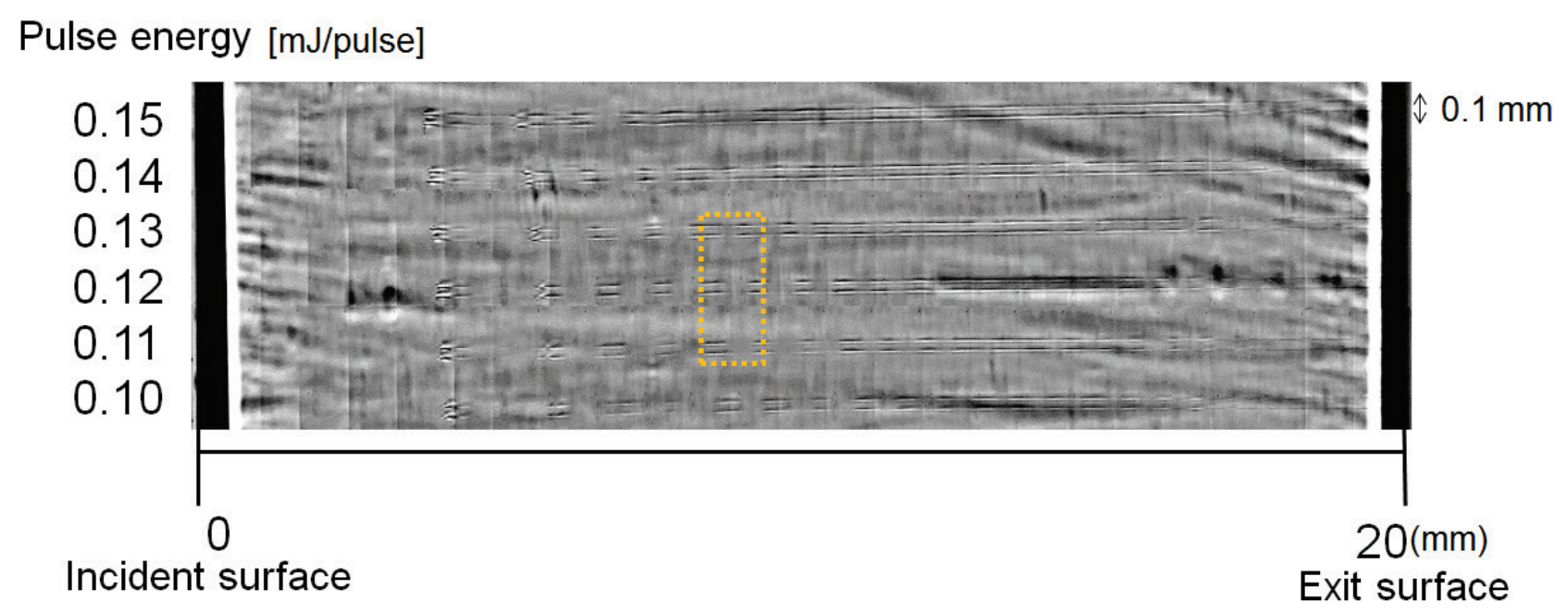

(a)

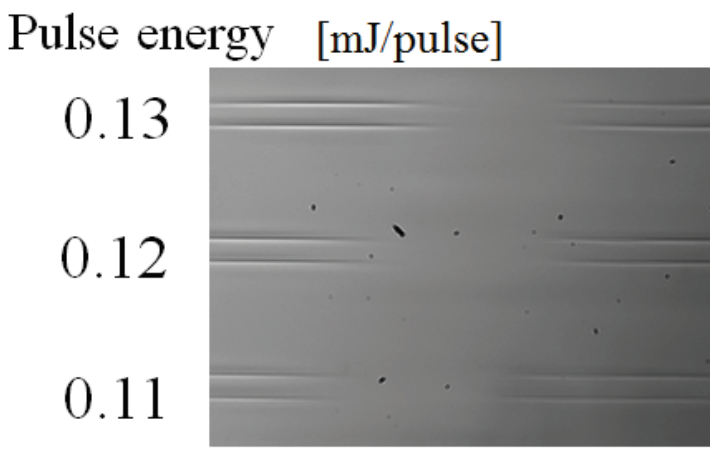

(b)

Fig. 8(a) Variations of structural modifications for different pulse energies at the distance $L=5 \mathrm{~mm}$. The sample was translated perpendicularly to the optical axis at $0.5 \mathrm{~mm} / \mathrm{s}$ perpendicular to the optical axis and repeated twice at the interval of $50 \mu \mathrm{m}$. (b) Magnified image of focusing and refocusing of filamentary modifications.

\section{Discussion}

Results show that the length of the filamentary modifications induced by the Bessel-Gaussian beam in PMMA was greater than that found with objective lenses. When laser pulses are focused in PMMA by objective lenses with a numerical aperture (NA) of 0.1 at pulse energy of a few microjoules per pulse, a filamentary refractive-index change at length of approximately $300 \mu \mathrm{m}$ was induced [16]. The length of modification with the axicon is substantially greater than that obtained by focusing a Gaussian beam. The aspect ratios of the structural modification are greater than 1,000 through processing with the axicon. Results show that filamentary modifications are induced in PMMA by focusing femtosecond laser pulses with an axicon at a cone angle of $170^{\circ}$. The axicon lens yields longer filamentary modifications, although higher energy is required.

Energy to induce filamentary modifications with the axicon requires greater than $0.1 \mathrm{~mJ} /$ pulse in PMMA. Femtosecond laser filamentation in glass and crystals with Bessel beams demands higher incident energy. Actually, energy of $0.6 \mathrm{~mJ} /$ pulse is necessary to generate filamentary modifications inside barium fluoride crystals and glass when an ax- icon is used. This result demonstrates that the threshold for the induction of modifications in PMMA is lower than that in glass or crystal.

Multiple filamentation has been observed when focusing a femtosecond laser pulse into a methanol solution with an axicon at a cone angle of $179^{\circ}$ [30]. Our results show that an axicon with a cone angle of $170^{\circ}$ produced multiple filaments at the beginning of propagation in PMMA. The transition between a single filament and multiple filaments might depend on the distance between the axicon tip, the sample surface, incident energy, and the cone angle of the axicon.

Our results obtained for focusing-refocusing filamentary tracks in PMMA show good agreement with findings in crystal and liquid [29-31]. When the laser energy increased, more refocusing filaments were created. Moreover, the periods between focusing and the refocusing cycles varied from $1.7 \mathrm{~mm}$ to $0.65 \mathrm{~mm}$. The periods decreased as the filament propagated. The length of focusing filamentary region varied from $1 \mathrm{~mm}$ to $0.3 \mathrm{~mm}$. At higher energies, focus and refocus tend to become mutually connected and longer filamentary channels were formed. The self-channeling 
mechanism relies on dynamic competition between selffocusing and plasma formation. Complex filamentary modifications were induced in PMMA.

\section{Conclusion}

We experimentally investigated filamentary structural modifications in PMMA by focusing femtosecond laser pulses with an axicon. Single and multiple filamentary modifications were induced in PMMA by focusing femtosecond laser pulses with an axicon at a cone angle of $170^{\circ}$. The region of filamentary modification was greater than $10 \mathrm{~mm}$ long. Focusing-refocusing modifications of filaments were produced, which is dependent on incident energies. The diffraction-free nature of Bessel beam with the axicon provides longer filamentary structural modifications. It is expected to be useful for internal modification and the photonic device fabrication in PMMA.

\section{Acknowledgments}

This work was supported by the Amada Foundation.

\section{References}

[1] R. R. Gattass and E. Mazur: Nature Photonics, 2, (2008) 219.

[2] K. Itoh, W. Watanabe, S. Nolte, and C. B. Schaffer: Mrs. Bulletin, 31, (2006) 620.

[3] Y. Shimotsuma, K. Hirao, P. G. Kazansky, and J. Qiu: Jpn. J. Appl. Phys., 44, (2005) 4735.

[4] G. Della Valle, R Osellame, and P. Laporta: J. Opt. A: Pure Appl. Opt., 11, (2009) 013001

[5] A. Couairon and A. Mysyrowicz: Phys Rep, 441, (2007) 47.

[6] L. Berg, S. Skupin, R. Nuter, J. Kasparian, and J.-P. Wolf: Rep. Prog. Phys., 70, (2007) 1633.

[7] K. Yamada, W. Watanabe, T. Toma, K. Itoh, and J. Nishii: Opt. Lett., 26, (2001) 19.

[8] W. Watanabe: Laser Physics, 19, (2009) 342.

[9] S. Sowa, W. Watanabe, T. Tamaki, J. Nishii, and K. Itoh: Opt. Express 14, (2006) 291.

[10] W. Watanabe, S. Sowa, T. Tamaki, K. Itoh, and J. Nishii: Jpn. J. Appl. Phys. Part 2, 45, (2006) L765.

[11] A. Zoubir, C. Lopez, M. Richardson, and K. Richardson: Opt. Lett., 29, (2004) 1840.

[12] S. Sowa, W. Watanabe, J. Nishii, and K. Itoh: Appl. Phys. A-Materials Science \& Processing, 81, (2005) 1587.

[13] P. J. Scully, D. Jones, and D. A. Jaroszynski: J. Opt. A-Pure and Applied Optics, 5, (2003) S92.

[14] A. Baum, P. J. Scully, W. Perrie, D. Jones, R. Issac, and D. A. Jaroszynski: Opt: Lett., 33, (2008) 651.

[15] A. Baum, P. J. Scully, W. Perrie, D. Liu, and V. Lucarin: J. Opt. Soc. Am. B, 27, (2010) 107.

[16] H. Mochizuki, W. Watanabe, R. Ezoe, T. Tamaki, Y. Ozeki, K. Itoh, M. Kasuya, K. Matsuda, and S. Hirono: Appl. Phy. Lett., 92, (2008) 091120.
[17] S. Hirono, M. Kasuya, K. Matsuda, Y. Ozeki, K. Itoh, H. Mochizuki, and W. Watanabe: Appl. Phy. Lett., 94, (2009) 241122.

[18] W. Watanabe, K. Matsuda, S. Hirono, and H. Mochizuki: J. Laser Micro Nanoeng, 7, (2012) 58.

[19] W. Watanabe, K. Matsuda, S. Hirono, and H. Mochizuki: J. Laser Micro Nanogeng, 7, (2012) 171.

[20] J. Amako, D. Sawaki, and E. Fujii: J. Opt. Soc. Am. B, 20, (2003) 2562.

[21] Y. Matsuoka, Y. Kizuka, and T. Inoue: Appl. Phys., A Mater. Sci. Process., 84, (2006) 423.

[22] D. McGloin and K. Dholakia: Contemporary Physics, 46, (2005) 15.

[23] M. Duocastella and C. B. Arnold: Laser Photonics Rev., 6, (2012) 607.

[24] J.H. McLeod: J. Opt. Soc. Am., 44, (1954) 592.

[25] G. Indebetouw: J. Opt. Soc. Am. A, 6, (1989) 150.

[26] J. Durnin, J. J. Miceli, Jr., and J. H. Eberly: Phys. Rev. Lett., 58, (1987) 1499.

[27] P. Polesana, M. Franco, A. Couairon, D. Faccio, and P. Di Trapani: Phys. Rev. A 77, 043814 (2008).

[28] S. Akturk, B. Zhou, M. Franco, A. Couairon, and A. Mysyrowicz: Opt. Commun., 282, (2009) 129.

[29] H. Polesana, D.e Faccio, P. Di Trapani, A. Dubietis, A. Piskarskas, A. Couairon, and M. A. Porrasigh: Opt. Express, 13, (2005) 6160.

[30] X. Sun, H.Gao, B. Zeng, S. Xu, W. Liu, Y. Cheng, Z. $\mathrm{Xu}$, and G. Mu: Opt. Lett., 37, (2012) 857.

[31] K. Dota, A. Pathak, J. A. Dharmadhikari, D. Mathur, and A. K. Dharmadhikari: Phys. Rev. A, 86, (2012) 023808 .

[32] O. G. Kosareva, A. V. Grigor'evskii, and V. P. Kandidov: Quantum Electronics, 35, (2005) 1013.

[33] A. Marcinkevičius, S. Juodkazis, S.Matsuo, V. Mizeikis, and H. Misawa: Jpn. J. Appl. Phys., 40, (2001) L1197.

[34] E. Gaižauskas, E. Vanagas, V. Jarutis, S. Juodkazis, V. Mizeikis, and H. Misawa: Opt. Lett., 31, (2006) 80.

[35] M. K. Bhuyan, P. K. Velpula, J. P. Colombier, T. Olivier, N. Faure, and R. Stoian: Appl. Phy. Lett., 104, (2014) 021107.

[36] B. Yalizay, T. Ersoy, B. Soylu, and S. Akturk: Appl. Phys. Lett., 100, (2012) 031104.

[37] F. Courvoisier, P.-A. Lacourt, M. Jacquot, M. K. Bhuyan, L. Furfaro, and J. M. Dudley: Opt. Lett., 34, (2009) 3163.

[38] M. K. Bhuyan, F. Courvoisier, P.-A. Lacourt, M. Jacquot, L. Furfaro, M. J. Withford, and J. M. Dudley: Opt. Express, 18, (2010) 566.

[39] M.K. Bhuyan, F. Courvoisier, H.S. Phing, O. Jedrkiewicz, S. Recchia, P. Di Trapani, and J. M. Dudley: Eur. Phys. J. Special Topics, 199, (2011) 101.

[40] J. A. Dharmadhikari, R. Bernard, A. K. Bhatnagar, D. Mathur, and A. K. Dharmadhikari: Opt. Lett., 38, (2013) 172.

[41] M. Mikutis, T. Kudrius, G. Ŝekys, D. Paipulas, and S. Juodkazis: Opt. Material Express, 3, (2013) 1862.

[42] W.-J. Tsai, C.-J. Gu, C.-W. Cheng and J.-B. Horng: Opt. Eng., 53, (2014) 051503. 